\title{
The exact binomial test between two independent proportions: A companion
}

\author{
Louis Laurencelle ${ }^{\mathrm{a} \otimes}(0)$ \\ ${ }^{\mathrm{a}}$ Université d’Ottawa
}

\begin{abstract}
This note, a summary in English of a former article in French (Laurencelle, 2017), presents an outline of the theory and implementation of an exact test of the difference between two binomial variables. Essential formulas are provided, as well as two executable computer programs, one in Delphi, the other in R.
\end{abstract}

Keywords $\square$ exact test of two proportions. Tools $\square$ Delphi, R.

Acting Editor $\because$ Denis Cousineau (Université d'Ottawa)

Reviewers

- No reviewer

\section{louis.laurencelle@gmail.com}

10.20982/tqmp.17.2.p076

\section{Introduction}

Let there be two samples of given sizes $n_{1}$ and $n_{2}$, and and numbers $x_{1}$ and $x_{2}$ of "successes" in corresponding samples, where $0 \leq x_{1} \leq n_{1}$ and $0 \leq x_{2} \leq n_{2}$. The purpose of the test is to determine whether the difference $x_{1} / n_{1}-x_{2} / n_{2}$ results from ordinary random variation or if it is probabilistically exceptional, given a probability threshold. Treatises and textbooks on applied statistics report a number of test procedures using some form of normal-based approximation, such as a Chi-squared test, various sorts of $z$ (normal) and $t$ (Student's) tests with or without continuity correction, etc. (Howell, 2017; Siegel \& Castellan, 1988; Sokal \& Rohlf, 1995). The reader may also be aware of Fisher's "exact probability test", which is indeed an exact probability test but not a test applicable to independent proportions, the rows $\left(n_{1}, n_{2}\right)$ and columns $\left(x_{1}, x_{2}\right)$ totals being both fixed. Liddell (1978) proposes a test with an exact probability calculation, but an incomplete one that uses a single point estimate of the parametric probability for the null hypothesis of the test.

The test procedure of Liddell (1978) exploits a point estimate of $\pi$, the probabilistic reference parameter, which is the maximum likelihood estimator for two binomial series, i.e., here, $\hat{\pi}=\left(x_{1}+x_{2}\right) /\left(n_{1}+n_{2}\right)$. Using this single estimate and following a suggestion of Kendall and Stuart (1977), Liddell lists all possible values of the two proportions and their differences, comparing these with the ob- served difference and then accumulating the probabilities of equal or more extreme differences.

Our proposed test, similar to that of Liddell (1978), uses the same procedure of enumerating the $\left(n_{1}+1\right) \times\left(n_{2}+1\right)$ possible differences, this time exploiting the integral domain (0..1) of the $\pi$ parameter in combination with an appropriate weighting function.

\section{Derivation of the exact test}

Let $R_{1}=\left(x_{1}, n_{1}\right)$ and $R_{2}=\left(x_{2}, n_{2}\right)$ be the results of two binomial observations, and their observed difference $d_{O}=x_{1} / n_{1}-x_{2} / n_{2}$, where $R_{1} \sim B\left(x_{1} \mid n_{1}, \pi_{1}\right), R_{2} \sim$ $B\left(x_{2} \mid n_{2}, \pi_{2}\right)$, with $\pi_{1}$ and $\pi_{2}$ being the two unknown binomial probability parameters. The null hypothesis of no parametric difference stipulates that $\pi_{1}=\pi_{2}=\pi_{0}$. Under the null hypothesis and applying a one-sided test, what is the probability that $d \geq d_{O}$ ?

The distribution of the random variable $d=y_{1} / n_{1}-$ $y_{2} / n_{2}\left(0 \leq y_{1} \leq n_{1}, 0 \leq y_{2} \leq n_{2}\right)$ is statistically conditioned on two factors: the size parameters $n_{1}$ and $n_{2}$, which are given, and the two observed proportions $x_{1} / n_{1}$ and $x_{2} / n_{2}$, with respect to which the likelihood of the underlying $\pi_{0}$ value will vary.

With any given value of $\pi_{0}$, the probability sought for is $P\left\{d \geq d_{O}\right\}=p\left(H_{0}\right)$ is :

$$
p\left(\pi_{0}\right)=\sum_{y_{1}, y_{2}} b\left(y_{1} \mid n_{1}, \pi_{0}\right) \times b\left(y_{2} \mid n_{2}, \pi_{0}\right),
$$


where $b(y \mid n, \pi)$ is the binomial probability given by $\left(\begin{array}{l}n \\ y\end{array}\right) \pi^{y}(1-\pi)^{n-y}$. The sum is computed using every value of $y_{1}$ and $y_{2}, 0 \leq y_{1} \leq n_{1}, 0 \leq y_{2} \leq n_{2}$, for which

$$
y_{1} / n_{1}-y_{2} / n_{2} \geq x_{1} / n_{1}-x_{2} / n_{2}
$$

(for a right-side difference test). However. as pointed out above, any value of $\pi_{0}$ is not as likely as another, considering the observed, real values of binomial variables $x_{1}$ and $x_{2}$. Indeed, the likelihood of a given $\pi_{0}$ value with respect to the observed data is simply

$$
L\left(\pi_{0} \mid x_{1}, n_{1} ; x_{2}, n_{2}\right)=\pi_{0}^{x_{1}+x_{2}} \pi_{0}^{n_{1}+n_{2}-x_{1}-x_{2}} .
$$

This function, akin to the Beta function (Johnson, Kotz, \& Balakrishnan, 1995), has here its maximum value at $\hat{\pi}=$ $\left(x_{1}+x_{2}\right) /\left(n_{1}+n_{2}\right)$, the so-called maximum likelihood estimate used in Liddell (1978) test procedure.

Our procedure, which encompasses the whole domain of $\pi_{0}$, consists in weighting the summation (1) by the likelihood function (3), resulting in the following formula (see Laurencelle, 2017, for more details):

$$
P\left\{d_{\text {random }} \geq d_{O}\right\}=\frac{(N+1) ! n_{1} ! n_{2} !}{(2 N+1) ! X !(N-X) !} \sum_{y_{1}, y_{2}} * \frac{\left(X+y_{1}+y_{2}\right) !\left(2 N-X-y_{1}-y_{2}\right) !}{y_{1} !\left(n_{1}-y_{1}\right) ! y_{2} !\left(n_{2}-y_{2}\right) !}
$$

where $N=n_{1}+n_{2}, X=x_{1}+x_{2}$, and the right-hand summation $\sum^{*}$ of (4) is restricted to $\left(y_{1}, y_{2}\right)$ pairs such that $y_{1} / n_{1}-y_{2} / n_{2} \geq x_{1} / n_{1}-x_{2} / n_{2}$ for a right-side difference test, and vice-versa for a left-side test.

In Listing 1 and Listing 2 are provided two computer codes, one for Pascal / Delphi language, the other for $\mathrm{R}$ language, which perform the computation of $p\left\{d_{\text {random }} \geq\right.$ $\left.d_{O}\right\}$. For a two-sided test, state the probability $\min (2 \times$ $p, 1), p$ begin likely to overflow $1 / 2$ due to the discrete nature of the distribution of $d$.

\section{Two examples}

Below are two data sets illustrating the results of formula (4) for the difference between two independent proportions, together with the results of some other test procedures. The classical Chi-squared and $z$-tests (without continuity correction) need not be presented. As for the Anscombe (1948), Chanter (1975) and Freeman and Tukey (1950) tests, here F-T, they are normal $z$ tests using Fisher's arcsine normalizing transformation of proportions, each offering an original variant. A full-scale study of these and other variants is coming soon ("The statistical treatment of proportions including analysis of variance, with examples", in French in this journal).

\section{Authors' note}

The author wishes to thank the Editor for his helpful and clarifying suggestions.

\section{References}

Anscombe, F. J. (1948). The transformation of poisson, binomial and negative-binomial data. Biometrika, 35, 246-254. doi:10.1093/biomet/35.3-4.246

Chanter, D. O. (1975). Modifications of the angular transformation. Journal of the Royal Statistical Society Series B (Applied Statistics), 24, 354-359. doi:10.2307/2347101

Freeman, M. F., \& Tukey, J. W. (1950). Transformations related to the angular and the square root. Annals of Mathematical Statistics, 21, 607-611. doi:10.1214/ aoms/1177729756

Howell, D. C. (2017). Fundamental statistics for the behavioral sciences (9th ed.) Washington: Cengage Learning.

Johnson, N. L., Kotz, S., \& Balakrishnan, N. (1995). Continuous univariate distributions (vol. 2) (2nd ed). New York: Wiley.

Kendall, M. G., \& Stuart, A. (1977). The advanced theory of statistics, vol. 1. New York.

Laurencelle, L. (2017). Le test binomial exact de la difference entre deux proportions et ses approximations / the exact binomial test between two proportions and its approximations. The Quantitative Methods for Psychology, 13(2), 141-147. doi:10.20982/tqmp.13.2.p141

Liddell, D. (1978). Practical tests of 2 ?2 contingency tables. The Statistician, 25, 295-304. doi:10.2307/2988087

Siegel, S., \& Castellan, N. J. (1988). Nonparametric statistics for the behavioral sciences. New York: McGraw-Hill.

Sokal, R. R., \& Rohlf, F. J. (1995). Biometry (3rd ed.) New York: Freeman. 
Table 1 - Two examples of computations

\begin{tabular}{cccccccccc}
\hline$x_{1} / n_{1}$ & $x_{2} / n_{2}$ & $p_{1}$ & $p_{2}$ & $d_{O}$ & $p_{\text {Exact }}$ & $p_{\chi^{2}}, p z$ & $p_{\text {Anscombe }}$ & $p_{\text {Chanter }}$ & $p_{F-T}$ \\
\hline $13 / 20$ & $15 / 29$ & 0.650 & 0.517 & 0.133 & 0.183 & 0.178 & 0.183 & 0.183 & 0.186 \\
$13 / 112$ & $89 / 473$ & $0.188^{*}$ & $0.116^{*}$ & 0.072 & 0.032 & 0.035 & 0.031 & 0.030 & 0.033 \\
\hline
\end{tabular}

Note. ${ }^{*}$ The programs being coded for right-tail extreme probability evaluation, the $\left(x_{1} / n_{1}\right)$ and $\left(x_{2} / n_{2}\right)$ data are automatically swapped so that $p_{1} \geq p_{2}$.

\section{Appendix: The programs}

\section{Listing 1: Original Pascal / Delphi program}

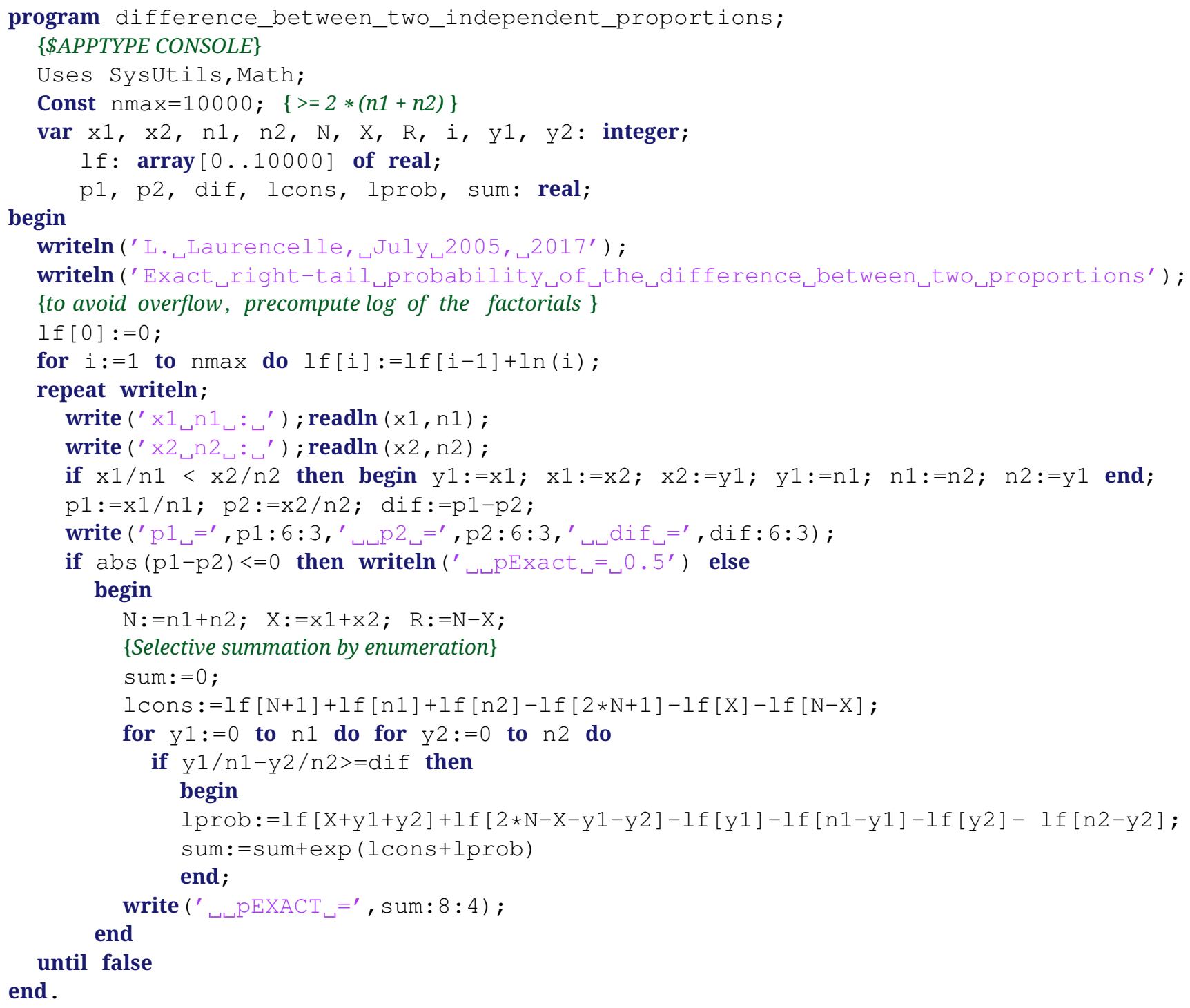




\section{Listing 2: $R$ implementation}

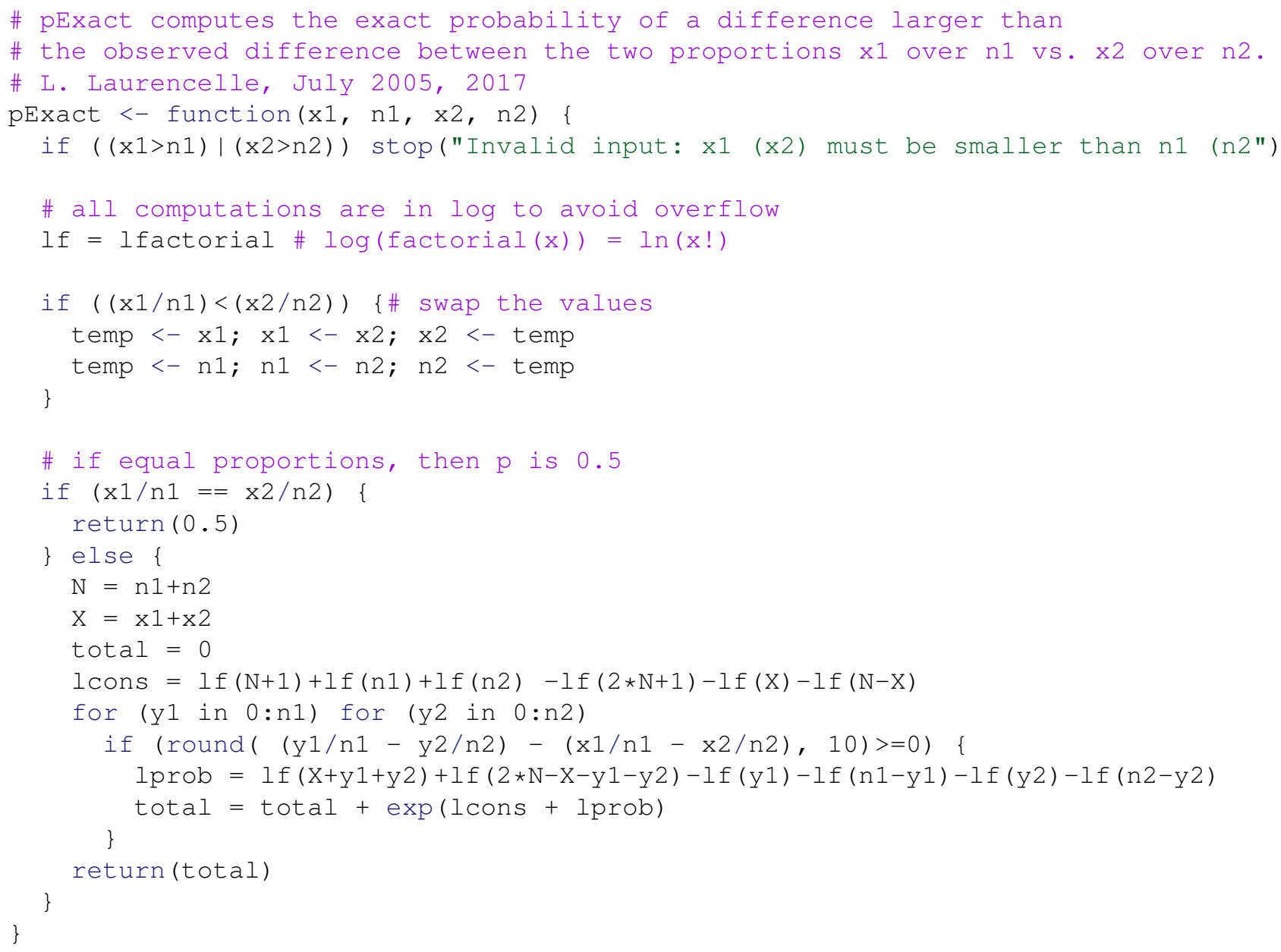

\section{Citation}

Laurencelle, L. (2021). The exact binomial test between two independent proportions: A companion. The Quantitative Methods for Psychology, 17(2), 76-79. doi:10.20982/tqmp.17.2.p076

Copyright $\odot$ 2021, Laurencelle. This is an open-access article distributed under the terms of the Creative Commons Attribution License (CC BY). The use, distribution or reproduction in other forums is permitted, provided the original author(s) or licensor are credited and that the original publication in this journal is cited, in accordance with accepted academic practice. No use, distribution or reproduction is permitted which does not comply with these terms.

Received: 07/05/2021 Accepted: 14/05/2021 